\title{
100 Jahre Coolidge-Röhre
}

\author{
Im Dezember 1913 wurde in Schenectady, New York die 1. \\ Hochvakuum-Röntgenröhre vorgestellt. Diese technische \\ Revolution war der Beginn einer neuen Ära in der Radiologie. \\ Damit wurde der Röntgenapparat zur technischen Maschine, \\ mit reproduzierbaren Bedingungen, bis heute.
}

Der Name Coolidge ist untrennbar mit einer bedeutenden technischen Entwicklung der Röntgenröhre verbunden. Seine Entwicklung der Glühkathoden-Hochvakuum-Röntgenröhre hat die Radiologie in

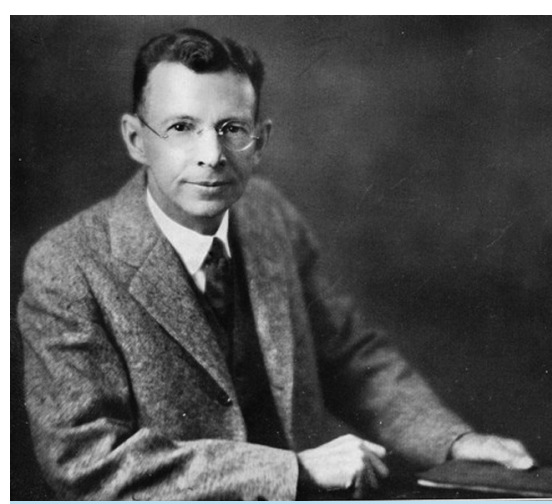

Coolidge Portrait (Quelle: Archiv Deutsches Röntgenmuseum).

Diagnostik und Therapie revolutioniert. Der US-amerikanische Röntgenpionier Lewis G. Cole (1874-1954) beschreibt Coolidge als einen Mann, der „außer Röntgen selbst mehr als alle anderen zur Förderung dieser Wissenschaft [die Radiologie] beigetragen hat." [1]

William David Coolidge wurde am 23.10.1873 auf einer kleinen Farm in der Nähe von Hudson, Massachusetts, USA, geboren. Nach dem Besuch der Public School erhielt er 1891 ein Stipendium am Massachusetts Institute of Technology (MIT). Schon als Student interessierte sich Coolidge für die Röntgentechnik und konstruierte bereits 1896 elektrostatische Generatoren zum Betrieb von IonenRöntgenröhren. Im gleichen Jahr erhielt er ein Auslandsstipendium. Coolidge entschied sich, seine Studien beim Leipziger Physiker Gustav Heinrich Wiedemann (1826-1899) fortzusetzen. In Leipzig promovierte er dann bei Paul Drude (18631906) mit einer Arbeit „Über eine neue Methode zur Demonstration elektrischer Drahtwellen“. Bevor Coolidge in die USA zurückkehrte, hatte er die Gelegenheit Wilhelm Conrad Röntgen persönlich kennenzulernen. Röntgen hatte einen Ruf nach Leipzig erhalten und besuchte am 26.11.1898 das physikalische Institut der Universität. Da Drude abwesend war, sollte Coolidge Röntgen empfangen. An seine Mutter schrieb er: „Zwar sieht er gar nicht so freundlich aus, dafür erscheint er sehr gewandt. Auf allen Bildern, die ich von ihm kenne, macht er einen schwindsüchtigen Eindruck, aber in Wirklichkeit ist er ein furchtbar großer und kräftiger Mann.“ [2]

Ab Herbst 1899 arbeitete Coolidge in den physikalisch-chemischen Laboratorien am MIT, bevor er 1905 in das Forschungslabor von General Electric in Schenectady, New York eintrat. 1932 wurde er Direktor des Forschungsinstitutes, dem er bis zu seinem Ausscheiden 1944 vorstand. Coo-

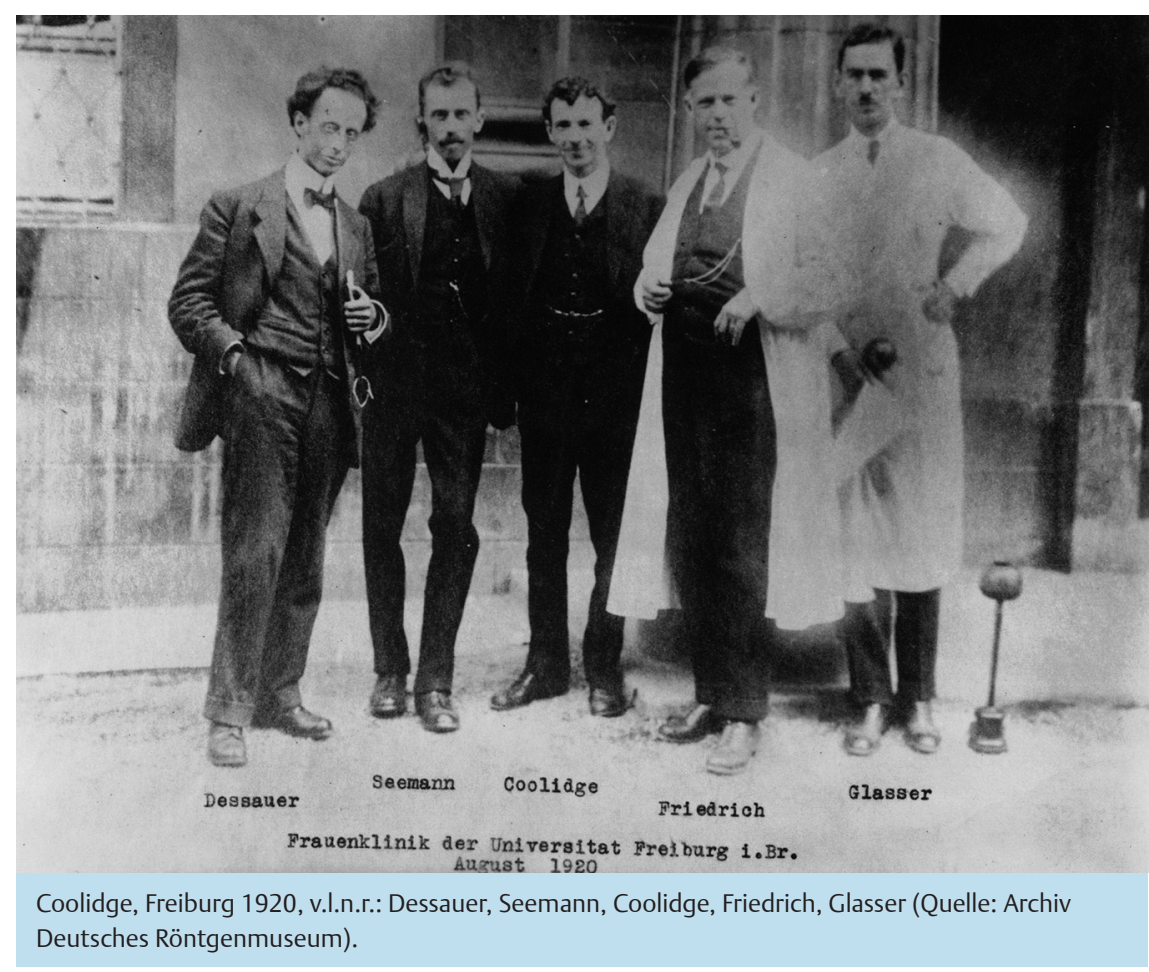

lidge veröffentlichte 80 wissenschaftliche Arbeiten und erhielt 83 Patente. Neben zahlreichen akademischen Auszeichnungen erhielt er 1963 die Röntgenplakette der Stadt Remscheid. Er verstarb am 3.2.1973 in Schenectady.

In Schenectady begannen auch seine epochemachenden Forschungen auf dem Gebiet der Wolfram-Metallurgie. Es gelang ihm als erster dünne Wolframdrähte herzustellen, die für elektrische Glühlampen verwendet werden konnten. Gestützt auf die erfolgreichen Versuche seines Vorgängers und damaligen Chefs und Nobelpreisträgers für Chemie 1932, Irving Langmuir (1881-1957), zur Elektronenemission glühender Wolframdrähte bei verschieden hohem Vakuum [3], entwickelte Coolidge 1913 nach knapp einjähriger Forschung die 1. „Röntgenröhre mit reiner Elektronenentladung“ [4]. Vorgestellt wurde die neue Entwicklung im Dezem- 


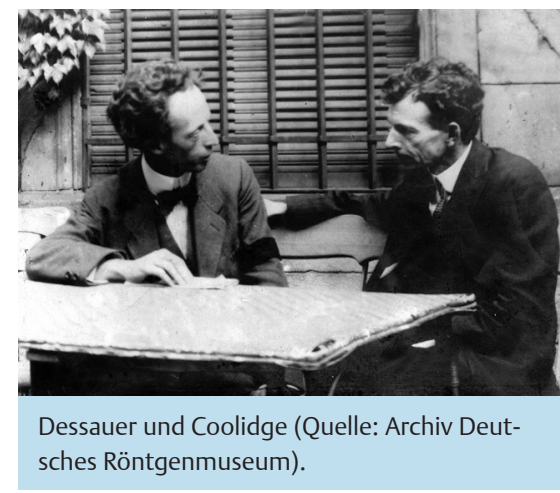

ber 1913. Cole taufte diese noch im selben Monat „Coolidge-Röhre“.

Den entscheidenden technischen Fortschritt, den die Coolidge-Röhre auslöste, beschrieb der Aschaffenburger Physiker und Röntgenpionier Friedrich Dessauer (1881-1963) wie folgt: „Die allergrößte Schwierigkeit bei der Konstruktion und beim Betrieb dar ,gashaltigen` Röhren bestand in dem Umstand, dass Strom- und Spannungsverlauf der Gasentladung hierbei nicht getrennt zu regeln sind. Erhöhung der Spannung bedeutet zugleich Vermehrung der Stromstärke und Änderung des Stromverlaufs. Die älteren Radiologen waren zum Teil recht gute Techniker und sie mussten es sein, um das Verhalten ihrer Röhren bei jedem Regulieren der

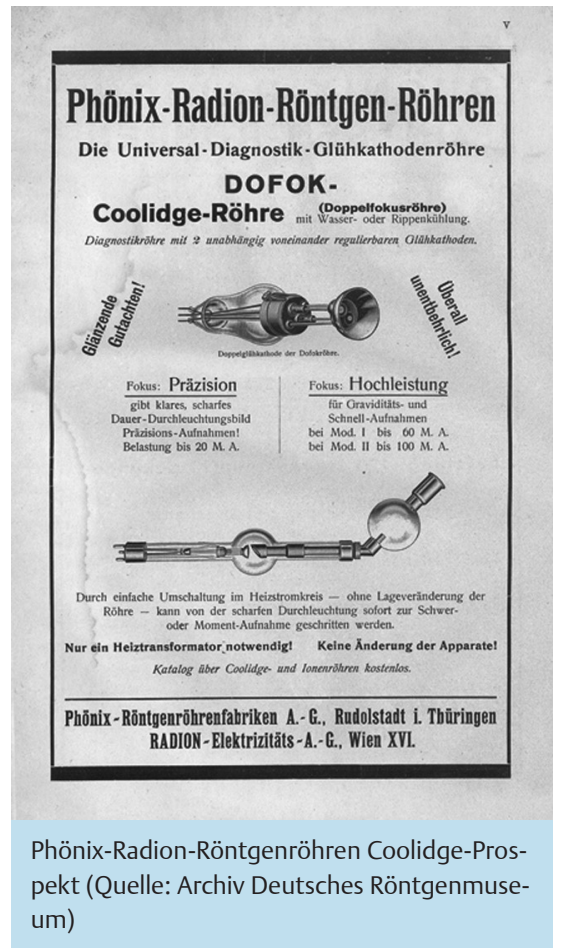

Spannungsquelle vorauszusehen, denn die Röhren waren sozusagen Individuen, von denen jede besonders reagierte. Diese Schwierigkeiten entfielen aufgrund der Erfindung der Coolidge-Röhre mit Hochvakuum und Glühkathode, die auch den
Einsatz des Funkeninduktors durch den Transformator zur Folge hatte. Damit wurde der Röntgenapparat zur technischen Maschine mit reproduzierbaren Bedingungen, bis heute.“ [5]

Nach ersten erfolgreichen Praxistests im Juli 1914 durch Heinrich Albers-Schönberg (1865-1921) übernahm die AEG in Berlin die Produktion der neuen Röntgenröhre in Deutschland. Ihr Siegeszug nahm seinen Lauf, genau wie Dessauer es 1956 beschrieb, bis heute.

\section{Dr. Uwe Busch,}

Deutsches Röntgenmuseum, Remscheid

\section{Literatur}

1 Cole LC, Vorläufige Mitteilung über die diagnostische und therapeutische Verwendung der Coolidge-Röhre. In: Fortschritte auf dem Gebiet der Röntgenstrahlen 22 (1914-15):29

2 Briefdokument WD Coolidge an seine Mutter, Leipzig 26.11.1898. Archiv Deutsches Röntgen-Museum

3 Langmuir I, Orange JA, Tungsten lamps of high efficiency. Gen. Elec. Rev. 16 (1913):956

4 W.D. Coolidge WD, The development of modern roentgen-ray generating apparatus. Am. J. Roentgenology. 24 (1930):605-620

5 Dessauer F, Erinnerungen aus der Entwicklung der Röntgentechnik. Röntgenblätter 9 (1956):70- 